\title{
Alteration of the Expression Levels of Rab3A Affects the Extent of Transcytosis of HRP-Labeled Marker Proteins in Rat CNS Neurons
}

\author{
Yoshiki Takeuchi $^{{ }^{*}}$, Yoshiki Matsumoto ${ }^{2}$, Takanori Miki ${ }^{1}$, Katsuhiko Warita ${ }^{1}$, Zhi-Yu Wang $^{1}$, \\ Kuldip S. Bedi ${ }^{3}$, Tomiko Yakura ${ }^{1}$, Jun-Qian Liu ${ }^{1}$
}

${ }^{1}$ Department of Anatomy and Neurobiology, Faculty of Medicine, Kagawa University, Kita-gun, Takamatsu, Japan; ${ }^{2}$ Department of Animal Science, Faculty of Agriculture, Kagawa University, Kita-gun, Takamatsu, Japan; ${ }^{3}$ Faculty of Health Sciences and Medicine, Bond University, Gold Coast, Australia.

Email: *yoshikit@med.kagawa-u.ac.jp

Received April 15 ${ }^{\text {th }}, 2011$; revised June 26 ${ }^{\text {th }}, 2011$; accepted July $30^{\text {th }}, 2011$.

\begin{abstract}
It has been hypothesized that Rab3A, a small GTPase, may be closely involved in the process of dense core vesicle exocytosis in various cell types. This possibility was investigated by disrupting the expression levels of Rab3A-mRNA using a small interfering RNA of the Rab3A GTPase (Rab3A-siRNA) and examining the effect of this on transcytosis of wheat germ agglutinin conjugated with horseradish peroxidase (WGA-HRP). Rab3A-siRNA and WGA-HRP were injected into the right vagus nerves of adult rats which were killed 12, 24 or 48 hours later. In some animals, portions of the brain stem containing the nucleus of solitary tract (NST) were prepared for electron microscopy. In other animals, the nodose ganglion of the vagus nerve was used to determine the levels of expression of Rab3A-mRNA using RT-PCR techniques. It was found that the expression of Rab3A-mRNA was markedly depressed in animals at $12 \mathrm{~h}$ after the Rab3A-siRNA injection. In the NST, there was an accumulation of HRP-reaction product $(R P)$, recognized as electron dense lysosomal-like structures, in both axons and terminals in the NST $12 \mathrm{~h}$ after injection. Some HRP-RP was found in membrane bound vesicles in close proximity to cell membranes and appeared to be in the process of transcytosis. This neuronal transcytosis of HRP-RP appeared to occur at random locations over the axodendritic membranes. These findings indicate that inhibiting the expression of Rab3A-mRNA using Rab3A-siRNA can modulate the level of transcytosis of proteins across neuronal membranes confirming the potentially important role of this GTPase in the process of transcytosis.
\end{abstract}

Keywords: Rab3A-siRNA, WGA-HRP, Transcytosis, Synapse, Vagus Nerve, Nucleus of Solitary Tract, Vesicle Docking, Quantitative Real Time RT-PCR

\section{Introduction}

Rab3A is a small GTP binding protein, associated with synaptic vesicles, that is considered to play an important role in modulation of synaptic transmitter release through regulation of vesicle trafficking and membrane fusion [15]. Rab3A also undergoes membrane dissociation-association, paralleling the synaptic membrane cycle, and is involved in regulating exocytosis as a synaptic vesicle membrane protein [6]. In a recent study using Rab3A gene-deleted mice, synaptic vesicle docking to the presynaptic membrane and regulation of synaptic vesicle exocytosis were shown to be impaired at the neuromuscular synapses $[7,8]$. Furthermore, our previous study in- dicated that Rab3A-siRNA injected into the rat vagus nerve is involved in modulating the level of transcytosis of the tracer enzyme, wheat germ agglutinin conjugated with horseradish peroxidase (WGA-HRP), at synaptic junctions [9]. However, the mechanisms involved in this effect of Rab3A-siRNA on transcytosis remain to be elucidated. The aim of the present study was to investigate the possible mechanisms involved.

\section{Materials and Methods}

The present experiments were performed on male Wistar rats (SLC, Hamamatsu, Japan), weighing $210-360$ g. The animals were housed in separate cages and maintained under standard laboratory conditions $\left(23^{\circ} \mathrm{C} \pm 1^{\circ} \mathrm{C}\right.$, 
12-h light: 12-h dark cycle, food and water ad libitum). The experimental procedures were conducted in accordance with National Institute of Health for Care and Use of Laboratory Animals. The Kagawa University Animal Care and Use Committee approved the procedures, and all efforts were made to minimize the number of animals used and their suffering.

Animals were anesthetized with intraperitoneal injection of chloral hydrate $(490 \mathrm{mg} / \mathrm{kg})$ for all surgical procedures. Groups of rats were each injected with 2.5 - 3.0 $\mu \mathrm{l}$ of a solution of $4 \%$ WGA-HRP alone (control group) or a solution of $4 \%$ WGA-HRP containing $1 \mathrm{nM}$ Rab3AsiRNA (experimental group) into the right vagus nerve using a $10-\mu l$ Hamilton micro-syringe. After a survival period of 12, 24 and $48 \mathrm{~h}$, the animals were sacrificed by perfusion through the ascending aorta with $0.1 \mathrm{M}$ phosphate buffer $(\mathrm{pH}$ 7.4) followed by a mixture of $1 \%$ paraformaldehyde and $1.25 \%$ glutaraldehyde in $0.1 \mathrm{M}$ phosphate buffer. The brain stem was removed from the skull. In 6 control and 7 experimental animals, $30 \mu \mathrm{m}$-thick frozen sections containing the nucleus of solitary tract (NST) and vagus verve nuclei were cut and processed for demonstration of HRP-reaction product (RP) according to the TMB method [10]. In another 12 control and 9 experimental animals, the blocks containing the NST and dorsal motor nucleus of the vagus nerve (DMV) were cut transversely into $200 \mu \mathrm{m}$-thick sections using a vibratome (Leica VT 1000S, Germany). These blocks were processed for demonstration of HRP-RP according to a heavy metal-intensified DAB method [11]. In experiments using the $\mathrm{DAB}$ method, the sections were postfixed in buffered $1 \%$ osmium tetroxide for $2 \mathrm{~h}$, block-stained in saturated uranyl acetate, dehydrated in alcohols and embedded in an epoxy resin mixture in preparation for electron microscopy. These blocks were cut on an ultramicrotome to yield $1 \mu \mathrm{m}$-thick sections which were stained with $1 \%$ toluidine blue. These sections were used to locate the regions of the block that contained the NST region. Ultrathin sections of this region were cut and observed without further lead staining using a JEM 200 CX electron microscope.

In addition, 9 control (non-operated), 15 WGA-HRP injection and 19 WGA-HRP injection with Rab3AsiRNA rats were perfused with RNase free condition of $0.9 \%$ saline after a survival period of $12 \mathrm{~h}$. The nodose ganglion ipsilateral to the injections was removed and quantitative real-time RT-PCR analysis of Rab3A-mRNA was performed. Total RNA was extracted from each nodose ganglion by homogenizing in TRIzol reagent (Invitrogen, USA). The concentration and purity of the extracted RNA were evaluated by optical density measurements at $260 \mathrm{~nm}$ and $280 \mathrm{~nm}$ using a spectrophotometer.

One microgram of total RNA in the nodose ganglion was dissolved in RNase-free water, mixed with reactionmix (including random primer and RNase inhibitor) and cDNA was synthesized using the High Capacity cDNA reverse transcription kit (Applied Biosystems, Foster City, USA). The cDNA was stored at $-80^{\circ} \mathrm{C}$ until comparison by Real-time quantitative RT-PCR analysis. To evaluate an appropriate internal control, co-amplification of a glyceraldehyde-3-phosphate dehydrogenase (GAPDH) mRNA was performed in each sample. The following forward (F) and reverse (R) primers used in the present study are shown in Table 1.

Quantitative real-time RT-PCR was performed using a LightCycler (Roche Diagnostics Ltd, Lewes, UK). Reactions for PCR were performed in a $20 \mu \mathrm{l}$ volume with 5 $\mu \mathrm{l}$ of the cDNA diluted 4 times, $0.5 \mu \mathrm{M}$ primers and reagents included in the LightCycler-FastStart DNA Master SYBR Green I mix (Roche Diagnostics Ltd, Lewes, UK). The amplification of cDNA protocol consisted of one cycle at $95^{\circ} \mathrm{C}$ for $10 \mathrm{~min}$ followed by 35 cycles at $95^{\circ} \mathrm{C}$ for $10 \mathrm{~s}, 65^{\circ} \mathrm{C}$ for $10 \mathrm{~s}, 72^{\circ} \mathrm{C}$ for $10 \mathrm{~s}$, and $87^{\circ} \mathrm{C}$ for $2 \mathrm{~s}$. Detection of the fluorescent products was carried out at the end of the $87^{\circ} \mathrm{C}$ extension period. To confirm amplification specificity, the PCR products were subjected to melting curve analysis. A similar electrophoresis of amplification product without reverse transcription (RT) was also performed for each sample as a negative control. The quantification of all data was analyzed with the LightCycler analysis software. Data on the mRNAs for Rab3A was expressed as the ratio of the mRNA for the housekeeping gene GAPDH (Table 1). Group means and

Table 1. Sequences of primer sets for quantitative real-time RT-PCR.

\begin{tabular}{ccc}
\hline & Forward primer & Reverse primer \\
\hline $\begin{array}{c}\text { Rab3A } \\
\text { (GenBank accession number: } \\
\text { X06889) }\end{array}$ & TGG CTG AGG CCA TCT TGC CC \\
$\begin{array}{c}\text { GAPDH } \\
\text { AB017801) }\end{array}$ & ACC ATC TAC CGC AAT GAC AAG A \\
$($ GenBank accession number: & GTA TTG GGC GCC TGG TCA CC & CGC TCC TGG AAG ATG GTG ATG G \\
\hline
\end{tabular}


standard errors were calculated for the three groups of animals. Data on Rab3A mRNA were analyzed by twoway analysis of variance (ANOVA) procedures. Post-hoc tests were carried out where appropriate using TukeyKramer's test [12]. All statistical analyses were carried out using SigmaStat (Systat Software, version 3.1) statistical software.

\section{Results}

\subsection{Light and Electron Microscopical Observations}

Injection of WA-HRP into the right vagus nerve resulted in heavy labeling of fibers in the NST dorsal or dorsolateral to the DMV with ipsilateral predominance. HRPRP was identified more clearly as electron-dense materials when lead citrate staining was omitted. In the neuropil of the NST, the RP showed membrane-bound lysosomes or lysosomal-like structures in axons and terminals. Although there was labeling present at all time periods studied after injection, labeling seemed to be more dense on the case of survival period of $48 \mathrm{~h}$. In addition, vesicle trafficking to active zones and docking to presynaptic membranes were observed in the terminals (Figure 2(a)). However, there were very few or no terminals showing neuronal transcytosis of HRP-RP.

Co-injection of Rab3A-siRNA with WGA-HRP also resulted in anterograde labeling which was more prominent in the NST ipsilateral to the injection side than in the contralateral side (Figure 1(a)). The labeling was greater 24 and $48 \mathrm{~h}$ after injection compared to $12 \mathrm{~h}$ after injection. The RP in the terminals, preterminals and axons was frequently observed to form a heavy accumulation (Figures 2(c)-(f)) and contain membranous substance showing structures which had the appearance of having been phagocytosed (Figure 2(e)). Approximately $60 \%$ of the total number of terminals $(n=214)$ containing the RP revealed not only suppression of vesicle docking to presynaptic membranes (Figures 2(b) and (c)) but also central concentration of synaptic vesicles containing small dense core vesicles (Figure 2(d)). Furthermore, it was of particular interest that the neuronal transcytosis of tracer enzyme appeared to have occurred randomly at various portions of the axodendritic membranes. The appearance of the HRP-RP in relation to the membranes was characterized as either the "passing type" (Figure 3(a)) or, because of the indentation of axodendritic membranes into the dendrites, as the "secretion type" (Figures 3(b)-(d)) [9].
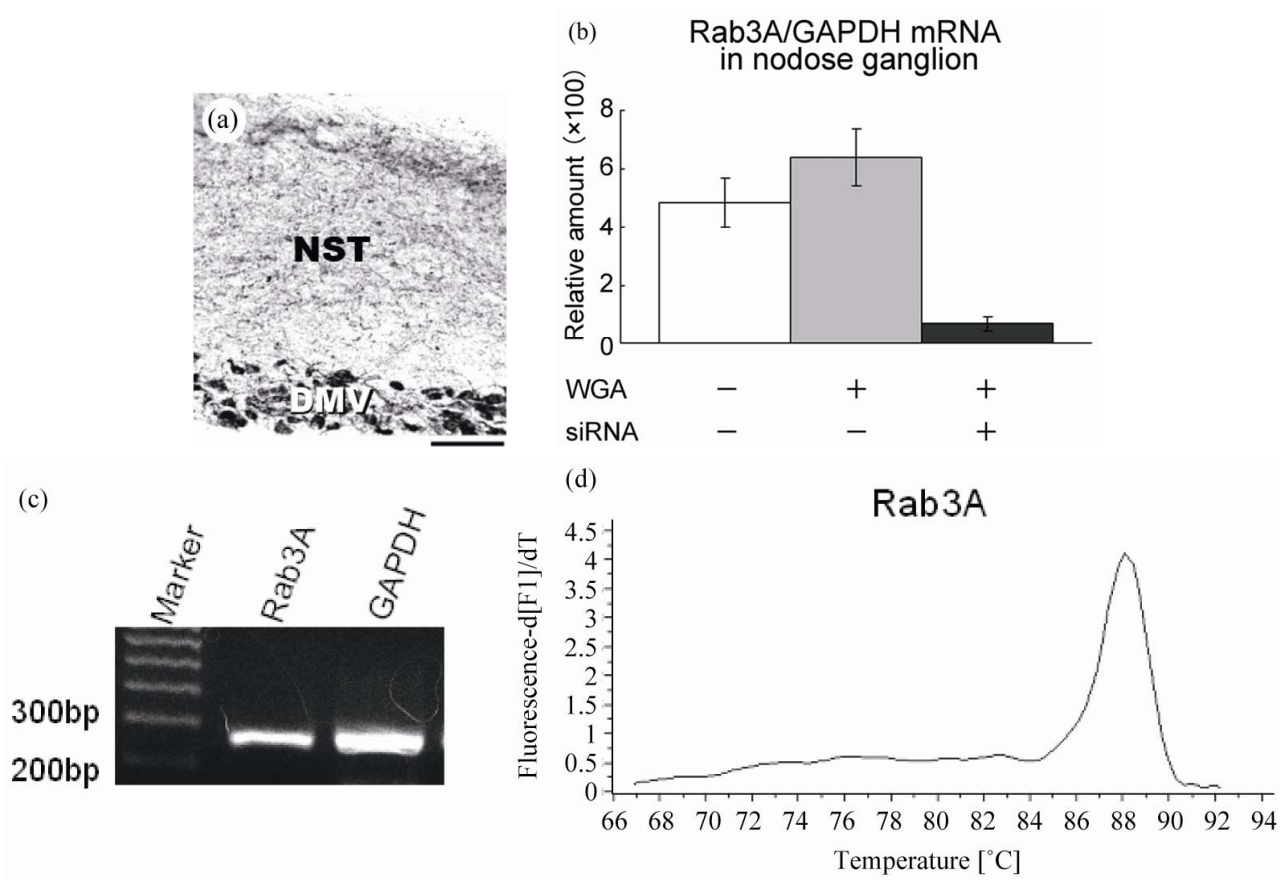

Figure 1. Takeuchi et al. Photomicrograph of labeling in the NST and DMV after co-injection of Rab3A-siRNA with WGAHRP into the vagus nerve (a), and quantitative real-time RT-PCR results of Rab3A- mRNA in the nodose ganglion after noninjected control, injection of WGA alone and co-injection with Rab3A-siRNA with WGA treatment at a survival period of 12 h (b). A representative agarose gel electrophoresis of PCR products of all groups of rats amplified from cDNA with RAb3A and GAPDH primers showing a single band (c) and single melting curve (d). Calibration bar $=100 \mu \mathrm{m}$ in A. DMV, dorsal motor nucleus of vagus nerve; NST, nucleus of solitary tract. 

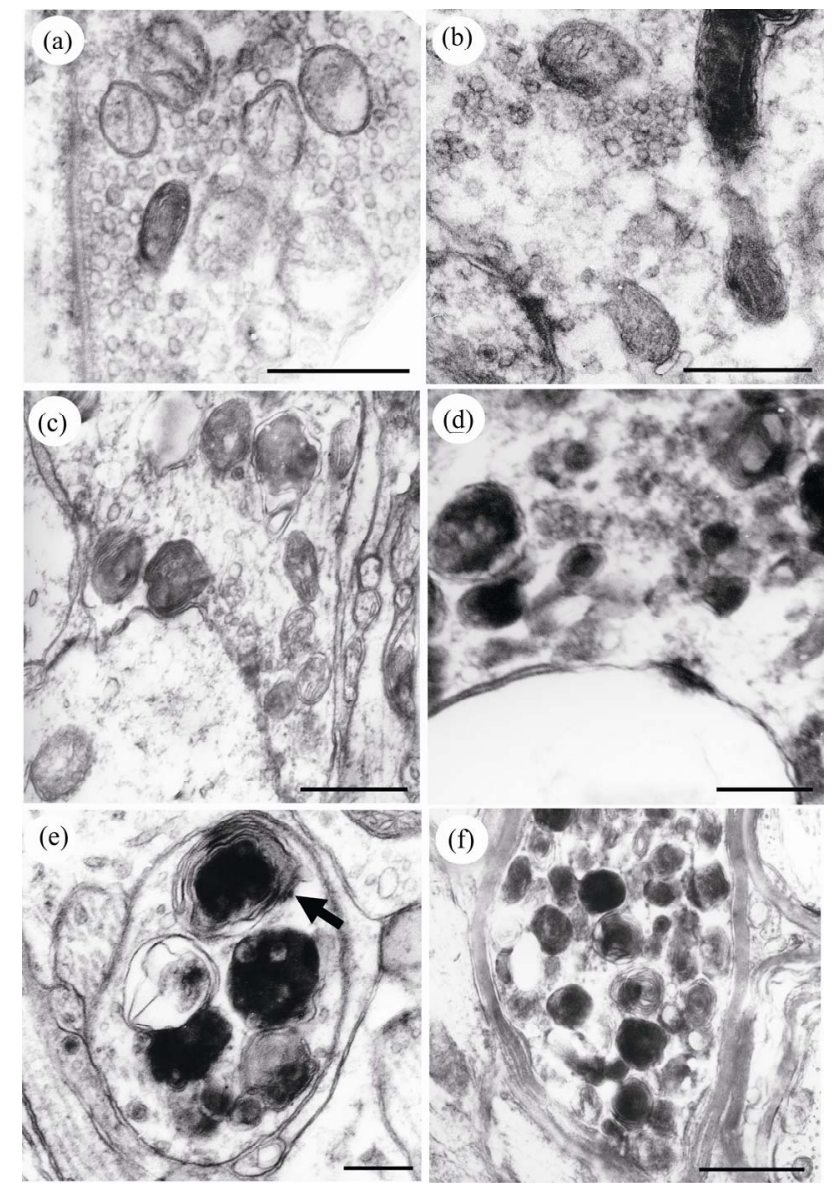

Figure 2. Takeuchi et al. Electron micrographs of HRP-RP in control (a) and experimental groups (b-f). The RP was identified more clearly as electron-dense materials when lead citrate staining was omitted. WGA-HRP injection resulted in a typical vesicle trafficking to active zones and docking to presynaptic membranes in the terminals (a), while co-injection of Rab3A-siRNA with WGA-HRP resulted in progressive accumulation of RP in terminals $(c, d)$, preterminals (e) and axons (f). Furthermore, co-injection showed not only suppression of vesicle docking (b-d) but also central concentration of synaptic vesicles containing small dense core vesicles (d). Arrow in $E$ indicates the appearance of phagocytosed RP. A survival period of 12 (f), 24 (d,e) and $48 \mathrm{~h}(\mathrm{a}-\mathrm{c})$. Calibration bars $=0.1 \mu \mathrm{m}$ in $(\mathrm{a}, \mathrm{b}, \mathrm{e}), 0.2$ $\mu \mathrm{m}$ in (c,d) and $1.0 \mu \mathrm{m}$ in (f).

\subsection{Quantitative RT-PCR Analysis}

The amplified PCR products from Rab3A and GAPDH showed a single and sharp transition in the melting curve analysis (Figure 1(d)) indicating a single PCR product. There was no evidence of primer-dimer formation with the number of cycles used in the present study. Electrophoresis of these PCR products showed a single band (Figure 1(c)). Omission of the cDNA synthesizing procedure resulted in there being no specific amplification

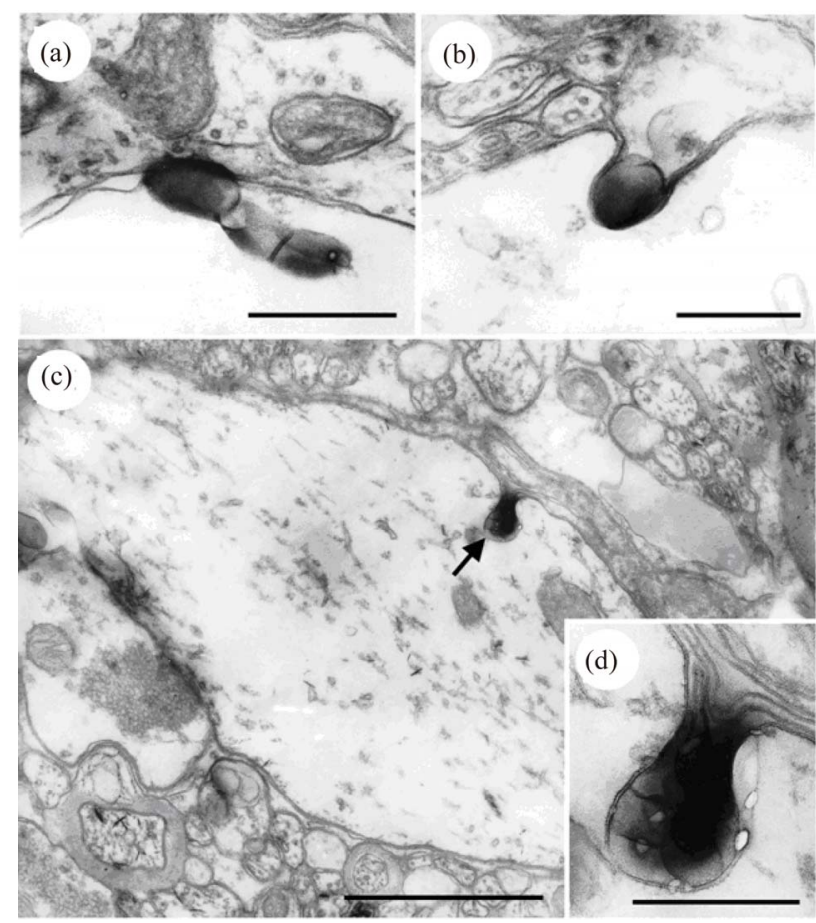

Figure 3. Takeuchi et al. Electron micrographs of "passing" (a) and "secretion" types (b-d) of neuronal transcytosis. The transcytosis of the RP was characterized by crossing the axodendritic membrane (a) and indentation of membranes into dendrites (b-d). Note that the neuronal transcytosis of tracer enzyme occurs randomly at various portions of axodendritic membranes. "Secretion" type on proximal dendrite in $C$ (arrow) is magnified in $D$. A survival period of 24 $h$ (a) and48 $h$ (b-d). Calibration bars $=0.3 \mu \mathrm{m}$ in $(a, b, d)$ and $1 \mu \mathrm{m}$ in (c).

of PCR products (data not shown). Figure 1(b) shows the mean \pm SEM ratio between the expressions of mRNA for Rab3A and the housekeeping gene GAPDH in the different groups of rats. Expression of Rab3A-mRNA in the nodose ganglion was apparently depressed after co-injection of Rab3A-siRNA with WGA-HRP into the vagus nerve, compared to that in control animals. Expression of Rab3A-mRNA in WGA-HRP injected group showed significantly higher measures than other two groups (Figure 1(b)). Rab3A selective siRNA mixture (target-specific siRNA for Rho/Rab subfamily, santa cruz, sc-36343) did not show any sign of behavioral toxicity.

\section{Discussion}

Our present study has shown that disruption of the expression of Rab3A using Rab3A-siRNA can modulate neuronal transcytosis as visualized using WGA-HRP labeling. The inhibition of the expression of Rab3A- mRNA in the nodose ganglion by the injection of Rab3A-siRNA into the vagus nerve was accompanied by morphological 
changes in the appearance of the neuronal terminals. There was a progressive accumulation of HRP-RP in axons and terminals. This accumulation might be caused by retardation of axonal transport to presynaptic membranes. Rab3A may be involved in axonal transport mechanisms. The morphological changes observed in the present study are consistent with the suggestion that there was a suppression of trafficking of HRP-labeled vesicles to active zones as well as inhibition of their docking to presynaptic membranes.

The "passing type" of transcytosis of protein demonstrated in the present and previous studies is quite similar to the diacrine manner in the exocrine gland which is frequently observed in secretion into the intestinal lumen [13]. In the central nervous system, the diacrine manner is also suggested to exist in GABA release during the cerebellar development [14]. On the other hand, "secretion type" shows the apocrine-like manner observed in the sweat and mammary glands. The exact reasons for changes of membranes, particularly exhibiting the indentation of axodendritic membranes into the dendrites in the rat co-injected with Rab3A-siRNA and WGA-HRP, are unclear. However, cytoskeletal changes of neurons might lead to the initiation of the "secretion" type of transcytosis because WGA and Rab proteins exert effects on cellular content of F-actin [15-17].

Neuronal transcytosis of protein has been well documented for neurotrophic factors, such as IGF, NT-3 and BDNF [18-21]. In those studies, transcytosis is described in detail and shown to require the initial packaging of the proteins involved into large dense-core vesicles (LDCVs) by the cellular Golgi system followed by the release of the neurotrophic factors from LDCVs at the nerve terminals. However, the findings reported here show the morphological characteristics of inter-neuronal transfer of protein without packaging into LDCVs. This apparently means that in transcytosis of proteins there is an independent system for chemical transmission induced by exocytosis of synaptic vesicles including LDCVs. In the present study the change of synaptic membranes induced by Rab3A-siRNA effects on the pattern of neuronal transcytosis of proteins which are increased in neuronal cells. It should be needed for such brain conditions to investigate the relationship with neurological disorder. In this point, our recent study in ethanol exposure rats showed "apocrine-like structure" which was quite similar to that reported here and additionally lower expression of Rab3A-mRNA in the specific areas of the central nervous system (unpublished data). On the other hand, coinjection of Rab3A-siRNA with WGA-HRP resulted in small DCVs in terminals as shown in Figure 2(d). This finding might raise the possibility that inhibition of the expression of Rab3A-mRNA leads to loss or reduction of size of DCVs and interferes with biogenesis of DCVs $[22,23]$.

The present study indicated that Rab3A-siRNA leads to morphological changes of membranes and is involved in regulating the neuronal transcytosis of protein. The morphology of the transcytosis of protein demonstrated here is quite different from vesicular exocytosis involved in chemical synaptic transmission.

\section{Acknowledgements}

The authors acknowledge the skillful technical assistance of Mr. Wakashi Nagata and Mrs. Mizue Fukutomi. This investigation was supported by the grant for science research from the Japanese Ministry of Education (No. 21791036 and 22591287).

\section{REFERENCES}

[1] A. Mizoguchi, S. Kim, T. Ueda, A. Kikuchi, H. Yorifuji, N. Hirokawa and Y. Takai, "Localization and Subcellular Distribution of smg p25A, a Ras p21-Like GTP-Binding Protein, in Rat Brain," The Journal of Biological Chemistry, Vol. 265, No. 20, 1990, pp. 11872-11879.

[2] M. Matteoli, K. Takei, R. Cameron, P. Hurbut, P. A. Johnston, T. C. Sudhof, R. Jahn and P. De Camilli, "Association of Rab3A with Synaptic Vesicles at Late Stages of the Secretory Pathway," The Journal of Cell Biology, Vol. 115, No. 3, 1991, pp. 625-633. doi: $10.1083 /$ jcb.115.3.625

[3] M. Geppert, V. Y. Bolshakov, S. A. Siegelbaum, K. Takei, P. De Camilli, R. E. Hammer and T. C. Sudhof, "The Role of Rab3A in Neurotransmitter Release," Nature, Vol. 369, No. 6480, 1994, pp. 493-497.

[4] O. M. Schlüter, F. Schmitz, R. Jahn, C. Rosenmund and T. C. Südhof, "A Complete Genetic Analysis of Neuronal Rab3 Function," The Journal of Neuroscience, Vol. 24, No. 29, 2004, pp. 6629-6637.

[5] M. S. Sons and J. J. Plomp, "Rab3A Deletion Selectively Reduces Spontaneous Neurotransmitter Release at the Mouse Neuromuscular Synapse," Brain Research, Vol. 1089, No. 1, 2006, pp. 126-134. doi:10.1016/j.brainres.2006.03.055

[6] J. Y. Li, R. Jahn and A. Dahlström, "Rab3a, a Small GTP-Binding Protein, Undergoes Fast Anterograde Transport but Not Retrograde Transport in Neurons," European Journal of Cell Biology, Vol. 67, No. 4, 1995, pp. 297307.

[7] A. G. Leenders, F. H. Lopes da Silva, W. G. Ghijsen and M. Verhage, "Rab3a Is Involved in Transport Of Synaptic Vesicles to the Active Zone in Mouse Brain Nerve Terminals," Molecular Biology of the Cell, Vol. 12, No. 10 , 2001, pp. 3095-3102.

[8] W. L. Coleman, C. A. Bill and M Bykhovskaia, "Rab3a Deletion Reduces Vesicle Docking and Transmitter Re- 
lease at the Mouse Diaphragm Synapse," Neuroscience, Vol. 148, No. 1, 2007, pp. 1-6.

[9] Y. Takeuchi, Y Matsumoto, T. Miki, T. Yokoyama, K. Warita, Z. Y. Wang, T. Ueno, T. Yakura and M. Fujita, "Anterograde Synaptic Transport of Neuronal Tracer Enzyme (WGA-HRP): Further Studies with Rab3A-siRNA in the Rat," Biomedical Research, Vol. 20, No. 3, 2009, pp. 149-154. doi:10.4103/0970-938X.54832

[10] M. M. Mesulam, "Tetramethylbenzidine for Horseradish Peroxidase Neurohistochemistry: A Non-Carcinogenic Blue Reaction Product with Superior Sensitivity for Visualizing Neural Afferents and Efferents," The Journal of Histochemistry and Cytochemistry, Vol. 26, No. 2, 1978, pp. 106-117. doi: 10.1177/26.2.24068

[11] A. Mizoguchi, S. Kim, T. Ueda, A. Kikuchi, H. Yorifuji, N. Hirokawa and Y. Takai, "Localization and Subcellular Distribution of Smg p25A, a Ras p21-like GTP-Binding Protein in Rat Brain," The Journal of Biological Chemistry, Vol. 265, No. 20, 1990, pp. 11872-11879.

[12] T. Miki, S. Harris, P. Wilce, Y. Takeuchi and K. S. Bedi, "The Effect of the Timing of Ethanol Exposure during Early Postnatal Life on Total Number of Purkinje Cells in Rat Cerebellum," Journal of Anatomy, Vol. 194, Pt. 3, 1999, pp. 423-431. doi:10.1046/j.1469-7580.1999.19430423.x

[13] J. Matsui, M. Fujimiya, S. Matsui, Y. Amakata, T. Renda, H. Kimura and T. Maeda, "Transient Expression of [DAla2] Deltorphin I-Like Immunoreactivity in Prenatal Rat Small Intestine," The Journal of Histochemistry and Cytochemistry, Vol. 42, No. 10, 1994, pp. 1377-1381. doi: $10.1177 / 42.10 .7930520$

[14] C. Takayama and Y. Inoue, "Extrasynaptic Localization of GABA in the Developing Mouse Cerebellum," Neuroscience Research, Vol. 50, No. 4, 2004, pp. 447-458.

[15] A. Sjölander and K. E. Magnusson, "Effects of Wheat Germ Agglutinin on the Cellular Content of Filamentous Actin in Intestine 407 cells," European Journal of Cell
Biology, Vol. 47, No, 1, 1988, pp. 32-35.

[16] C. E. Chua, Y. S. Lim and B. L. Tang, "Rab35-A Vesicular Traffic-Regulating Small GTPase with Actin Modulating Roles," FEBS Letters, Vol. 584, No. 1, 2010, pp. 1-6. doi:10.1016/j.febslet.2009.11.051

[17] M. van der Heijden, A. M. Versteilen, P. Sipkema, G. P. van Nieuw Amerongen, R. J. Musters and A. B. Groeneveld, "Rho-Kinase-Dependent F-Actin Rearrangement Is Involved in the Inhibition of PI3-kinase/Akt during Ischemia-Reperfusion-Induced Endothelial Cell Apoptosis," Apoptosis, Vol. 13, No. 3, 2008, pp. 404-412.

[18] C. S. von Bartheld, M. R. Bayers, R. Williams R and M. Bothwell, "Anterograde Transport of Neurotrophins and Axodendritic Transfer in the Developing Visual System," Nature, Vol. 379, No. 6568, 1996, pp. 830-833.

[19] X. G. Luo, R. A. Rush and X. F. Zhou, "Ultrastructural Localization of Brain-Derived Neurotrophic Factor in Rat Primary Sensory Neurons," Neuroscience Research, Vol. 39, No. 4, 2001, pp.377-384. doi:10.1016/S0168-0102(00)00238-8

[20] X. Wang, R. Butowt, M. R. Vasko and C. S. von Bartheld, "Mechanisms of the Release of Anterogradely Transported Neurotrophin-3 from Axon Terminals," The Journal of Neuroscience, Vol. 22, No. 3, 2002, pp. 931-945.

[21] C. S. von Bartheld, "Axonal Transport and Neuronal Transcytosis of Trophic Factors, Tracers, and Pathogens," Journal of Neurobiology, Vol. 58, No. 2, 2004, pp. 295314. doi:10.1002/neu.10315

[22] Y. Kim, J. H. T. Cheng, L. E. Eiden and Y. P. Loh, "Chromogranin A, an "on/off" Switch Controlling DenseCore Secretory Granule Biogenesis," Cell, Vol. 106, No. 4, 2001, pp. 499-509.

[23] H. Koshimizu, T. Kim, N. X. Cawley and Y. P. Loh, "Chromogranin A: A New Proposal for Trafficking, Processing and Induction of Granule Biogenesis," Regulatory Peptides, Vol. 160, No. 1-3, 2010, pp. 153-159. 